\title{
The Role of Dobutamine Dose on the Cardiac Parameters
}

\section{Rabindra Nath Das*}

Department of Statistics, The University of Burdwan, Burdwan 713104, West Bengal, India

\begin{abstract}
Objectives: The report presents the effects of dobutamine dose on the cardiac parameters such as blood pressures (basal, systolic, diastolic and maximum), heart rates (basal, peak and maximum), baseline cardiac ejection fraction, and ejection fraction on dobutamine dose. events.

Background: There is a little literature about the effects of dobutamine dose on the cardiac parameters and

Materials and Methods: The effects of dobutamine dose on the cardiac parameters have been examined based on a real echocardiography stress data set, collected at University of California, Los Angeles on 558 patients with 31 explanatory variables/factors. The distribution of the considered cardiac parameters is gamma with non-constant variance. So, the cardiac parameters have been analyzed by joint generalized linear gamma models.

Results: The mean basal blood pressure (BBP) decreases as the double product of maximum heart rate (MHR) and maximum blood pressure (MBP) at dobutamine dose (DPMAXDO) $(P<0.0001)$ increases, while the variance of BBP increases as the DPMAXDO $(P=0.0008)$ increases. The mean systolic blood pressure (SBP) increases as the dobutamine dose (DOSE) $(P=0.0268)$ increases, while the mean SBP increases as the DPMAXDO $(P<0.0001)$ decreases. The mean MBP increases as the DPMAXDO $(P<0.0001)$ increases. The mean baseline cardiac ejection fraction $(B E F)$ decreases as the DOSE $(P=0.0255)$ increases. The mean ejection fraction on dobutamine dose (DOBEF) increases as the DOSE $(P=0.0110)$ increases, while the variance of DOBEF increases as the dobutamine dose at maximum double product (DOBDOSE) $(P=0.0015)$ decreases. The mean basal heart rate $(\mathrm{BHR})$ increases as the DPMAXDO $(P<0.0001)$, or DOBDOSE $(P=0.0740)$ decreases. The mean peak heart rate $(P H R)$, or maximum heart rate $(M H R)$ increases as the DPMAXDO $(P<0.0001)$ increases, while the variance of PHR $(M H R)$ increases as the DOBDOSE $(P<0.0001)$ decreases (increases). On the other hand, dobutamine dose is associated with many cardiac parameters such as SBP, MBP, new myocardial infraction (new MI), history of MI (hxofMl) etc.
\end{abstract}

Conclusion: Only the marginal effects of dobutamine dose are observed on SBP, MBP, DOBEF, newMI, histMI, etc, while the joint effects of dobutamine dose (i.e., DPMAXDO and DOBDOSE) are observed on each cardiac parameters. The results are new inputs in the dobutamine dose study literature.

Keywords: Blood pressures; Cardiac parameters; Dobutamine dose; Ejection fractions; Heart rates; Joint gamma models

\section{Introduction}

It is well-known that myocardial $\beta_{1}$-adrenergic receptors are directly stimulated by dobutamine which is a synthetic catecholamine [1-3]. It is widely used to improve ventricular performance and myocardial contractility in patients with decompensated congestive heart failure or acute left ventricular dysfunction [4-6]. Generally, dobutamine effects on hemodynamics and cardiac mechanics, as a result, myocardial oxygen utilization $\left(\mathrm{MVO}_{2}\right)$ is altered in many ways. By increasing heart rate and contractility, $\mathrm{MVO}_{2}$ may be increased. For examining the patients with or without known cardiac disease having ischemia, practically, dobutamine stress echocardiography (DSE) is continuously, widely and successfully applied [7-10]. When the patients are unable to exercise by bicycle or treadmill to a certain degree for obtaining the necessary clinical information, DSE is used. As a result of applying DSE to the patients, echocardiographer has sufficient time to collect the required stress images at all levels.

Due to dobutamine use, there are several changes in the cardiac functions without significant changes in blood pressure, ventricular irritability, heart rate [8-13]. There are many cardiac parameters such as basal blood pressure, systolic blood pressure, diastolic blood pressure, maximum blood pressure, basal heart rate, peak heart rate, maximum heart rate, baseline cardiac ejection fraction, ejection fraction on dobutamine dose, etc. The present report focuses the role of dobutamine dose on the above cardiac parameters based on a real echocardiography stress data set, collected at University of California, Los Angeles on 558 patients with 31 explanatory variables/factors [14].

\section{Materials and Methods}

The considered echocardiography stress data set, patient population, data collection method are clearly displayed $[15,16]$, (Table 1). The factors/variables in the echocardiography stress data set are basal blood pressure (BBP), basal heart rate (BHR), basal double product (DP) of $\mathrm{BBP}$ and $\mathrm{BHR}(\mathrm{BDP})$, systolic blood pressure (SBP), peak heart rate (PKHR), DP of SBP and PKHR (DP), maximum heart rate (MHR), dobutamine dose (DD) given (DOSE), maximum blood pressure (MBP), DP of MHR and MBP at DD (DPMAXDO), percent maximum predicted heart rate (PMPHR), DD at max double product (DOBDOSE), sex (male=0, female=1) (SEX), age (AGE), ejection fraction on dobutamine (DOBEF), baseline cardiac ejection fraction (BEF), resting wall motion abnormality on echocardiogram

${ }^{*}$ Corresponding author: Rabindra Nath Das, Professor, Department of Statistics, The University of Burdwan, Burdwan 713104, West Bengal, India, Tel: +91 9232638970; E-mail: rabin.bwn@gmail.com

Received March 05, 2018; Accepted March 22, 2018; Published March 29, 2018

Citation: Das RN (2018) The Role of Dobutamine Dose on the Cardiac Parameters Cardiovasc Pharm Open Access 7: 238. doi: 10.4172/2329-6607.1000238

Copyright: (c) 2018 Das RN. This is an open-access article distributed under the terms of the Creative Commons Attribution License, which permits unrestricted use, distribution, and reproduction in any medium, provided the original author and source are credited. 
Citation: Das RN (2018) The Role of Dobutamine Dose on the Cardiac Parameters . Cardiovasc Pharm Open Access 7: 238. doi: 10.4172/23296607.1000238

Page 2 of 5

\begin{tabular}{|c|c|c|c|c|c|}
\hline Response & Covariate & Estimate & S.E & t-value & P-value \\
\hline \multirow{3}{*}{ Mean of dobutamine dose (DD) } & DP & -0.0001 & 0.000002 & -7.881 & $P<0.0001$ \\
\hline & DPMAXDO & 0.0001 & 0.000002 & 7.66 & $P<0.0001$ \\
\hline & DOBDOSE & 0.0227 & 0.000509 & 44.681 & $P<0.0001$ \\
\hline \multirow{8}{*}{ Variance of dobutamine dose (DD) } & SBP & -0.0463 & 0.0075 & -6.1381 & $\mathrm{P}<0.0001$ \\
\hline & DOBDOSE & -0.2704 & 0.0122 & -22.096 & $P<0.0001$ \\
\hline & SEX & -0.2693 & 0.13 & -2.072 & $P=0.0190$ \\
\hline & New MI & -0.9371 & 0.302 & -3.103 & $P=0.010$ \\
\hline & DEATH & -0.5704 & 0.336 & -1.698 & $P=0.0730$ \\
\hline & MBP & 0.0415 & 0.0076 & 5.446 & $\mathrm{P}<0.0001$ \\
\hline & posSE & 0.3642 & 0.1459 & 2.496 & $P=0.0110$ \\
\hline & Hxof MI & 0.2613 & 0.1438 & 1.818 & $P=0.0960$ \\
\hline Mean of basal BP (BBP) & DPMAXDO & -0.0001 & $<0.0001$ & -3.981 & $P<0.0001$ \\
\hline Variance of BBP & DPMAXDO & 0.0001 & $<0.0001$ & 3.372 & $P=0.0008$ \\
\hline \multirow{2}{*}{ Mean of SBP } & DOSE & 0.0005 & 0.0002 & 2.221 & $P=0.0268$ \\
\hline & DPMAXDO & -0.0001 & $<0.0001$ & -23.981 & $P<0.0001$ \\
\hline Mean of MBP & DPMAXDO & 0.0001 & $<0.0001$ & 59.712 & $P<0.0001$ \\
\hline Mean of BEF & DOSE & -0.0009 & 0.0004 & -2.241 & $\mathrm{P}=0.0255$ \\
\hline Mean of DOBEF & DOSE & 0.001 & 0.0004 & 2.551 & $P=0.0110$ \\
\hline Variance of DOBEF & DOBDOSE & -0.0217 & 0.0068 & -3.192 & $P=0.0015$ \\
\hline \multirow{2}{*}{ Mean of BHR } & DPMAXDO & 0 & 0.0001 & -3.671 & $P<0.0001$ \\
\hline & DOBDOSE & -0.0003 & 0.0001 & -1.792 & $P=0.0740$ \\
\hline Mean of PKHR & DPMAXDO & 0.0001 & 0.0001 & 6.392 & $P<0.0001$ \\
\hline Variance of PKHR & DOBDOSE & -0.0566 & 0.0084 & -6.765 & $\mathrm{P}<0.0001$ \\
\hline Mean of MAXHR & DPMAXDO & 0.0001 & 0.0001 & 13.052 & $P<0.0001$ \\
\hline Variance of MAXHR & DOBDOSE & 0.0243 & 0.0066 & 3.698 & $P<0.0010$ \\
\hline
\end{tabular}

Table 1: Results of the role of dobutamine dose on cardiac parameters.

(ECDG) $($ yes $(y)=0$, no $(n)=1)($ RESTWMA $)$, chest pain $(y=0, n=1)$ (CHESTPAIN), new myocardial infraction (MI) $(\mathrm{y}=0, \mathrm{n}=1)$ (new MI), positive stress on ECDG $(\mathrm{y}=0, \mathrm{n}=1)$ (posSE), recent bypass surgery $(\mathrm{y}=0, \mathrm{n}=1)$ (new CABG), recent angioplasty $(\mathrm{y}=0, \mathrm{n}=1)$ (new PTCA), death $(y=0, n=1)(D E A T H)$, history of diabetes $(y=0, n=1)($ hxof DM), history of hypertension $(\mathrm{y}=0, \mathrm{n}=1$ ) (hxof HT), history of angioplasty $(\mathrm{y}=0, \mathrm{n}=1)$ (hxof PTCA), history of smoking (non-smoker $=0$, moderate $=1$, heavy=2) (hxof Cig), history of MI ( $y=0, n=1)$ (hxof MI), history of coronary artery bypass surgery $(y=0, n=1)$ (hxof CABG), baseline electrocardiogram diagnosis (normal $=0$, equivocal $=1, \mathrm{MI}=2$ ) (ECG), death, new MI, new PTCA or new CABG (death=0, no=1) (EVENT).

\section{Statistical methods}

The considered study responses (BBP, SBP, MBP, BHR, PHR, MHR, BEF, DOBEF) are all positive, heteroscedastic, and gamma distributed. These responses are analyzed by gamma joint generalized linear models. Note that positive, heteroscedastic and continuous responses should be analyzed by joint generalized linear Log-normal or gamma models. Detailed discussions of these two models are given [16-19], which is not reproduced in the report. Very shortly, only the joint generalized linear gamma models (appropriate for these responses) are described herein.

For the positive response random variable $y_{i}$ 's, $E\left(y_{i}\right)=\mu_{i}$ and Var $\left(\mathrm{Y}_{\mathrm{i}}\right)=\sigma_{i}^{2} \mu_{\mathrm{i}}^{2}$, where $E\left(y_{i}\right)=\mu_{i}$ is the mean parameter, and $\sigma_{i}^{2}$ is the dispersion parameter. Generally, the variance function in generalized linear models (GLMs) is denoted by $V(\cdot)$, which has two components. First component is $\sigma_{i}^{2}$, which does not depend on the mean changes, and the second component is $V\left(\mu_{i}\right)$, which depends on the mean adjustment. Note that the GLM family distribution is identified by the variance function. For example, if $V(\mu)=\mu^{2}$, the distribution is gamma. Similarly, the distribution is Poisson, or Normal according as $V(\mu)=\mu$, or $V(\mu)=1$.

The joint GLMs (JGLMs) for the mean and dispersion parameters are:

$$
\eta_{i}=g\left(\mu_{i}\right)=x_{i}^{t} \beta \text { and } \varepsilon_{i}=h\left(\sigma_{i}^{2}\right)=w_{i}^{t} \gamma,
$$

Where $x_{i}^{t}, w_{i}^{t}$ are the row vectors of independent factors/variables for regression models, and $g(\cdot)$ and $h(\cdot)$ are the generalized linear model link functions (a function between the mean and the variance with the linear predictors). Maximum likelihood (ML) method is used to estimate the mean parameters $(\beta)$, and the restricted ML method is applied to estimate the dispersion parameters $(\gamma)[16,17]$.

The current report focuses the effects of dobutamine dose on the cardiac parameters such as basal blood pressure, systolic blood pressure, maximum blood pressure, basal heart rate, peak heart rate, maximum heart rate, baseline cardiac ejection fraction, ejection fraction on dobutamine dose. The above stress data set does not contain diastolic blood pressure. So, it is not possible to locate the effects of dobutamine dose on diastolic blood pressure for the above data set. The effect of dobutamine dose on a cardiac parameter can be derived by an appropriate probabilistic modeling of the targeted cardiac parameter on the dobutamine dose, and along with the remaining explanatory variables/factors. On the other hand, by deriving an appropriate probabilistic modeling of the dobutamine dose on the cardiac parameters and along with the remaining explanatory variables/factors, the probabilistic models of basal blood pressure, systolic blood pressure, and maximum blood pressure are given [15]. The probabilistic models of basal heart rate, peak heart rate, and maximum heart rate are given [20], and also the probabilistic models of baseline cardiac ejection fraction and ejection fraction on 
Citation: Das RN (2018) The Role of Dobutamine Dose on the Cardiac Parameters . Cardiovasc Pharm Open Access 7: 238. doi: 10.4172/23296607.1000238

dobutamine dose are given [21]. The joint (mean and variance) probabilistic models of dobutamine dose on the cardiac parameters, and along with the remaining explanatory variables/factors have been derived herein (Table 1).

\section{Effects of dobutamine dose on blood pressures (based on blood pressure models)}

The effects of dobutamine dose on blood pressures (basal, systolic and maximum) are derived [15]. In [15], the probabilistic models of basal blood pressure, systolic blood pressure, and maximum blood pressure are given. For ready reference, the necessary results related to dobutamine dose are reproduced in. From [16], it is observed that (i) mean basal blood pressure (BBP) is inversely correlated with the double product of MHR and MBP at dobutamine dose (DPMAXDO) $(\mathrm{P}<0.0001)$, indicating that the mean BBP increases as the DPMAXDO decreases. (ii) Variance of $\mathrm{BBP}$ is directly correlated with the DPMAXDO $(\mathrm{P}=0.0008)$, indicating that $\mathrm{BBP}$ variance increases as the DPMAXDO increases. These results are given in Table 1. From [15], it is observed that (i) mean systolic blood pressure (SBP) is directly correlated with the dobutamine dose (DOSE) $(\mathrm{P}=0.0268)$, indicating that SBP increases as the DOSE increases. In addition, (ii) the mean SBP is inversely correlated with the DPMAXDO $(\mathrm{P}<0.0001)$, indicating that SBP increases as the DPMAXDO decreases. From [16)], it is observed that (i) mean of maximum blood pressure (MBP) is directly correlated with the DPMAXDO $(\mathrm{P}<0.0001)$, indicating that MBP increases as the DPMAXDO increases. These results are given in Table 1.

\section{Effects of dobutamine dose on cardiac ejection fractions (based on ejection fraction models)}

The effects of dobutamine dose on cardiac ejection fractions (baseline cardiac ejection fraction and cardiac ejection fraction on dobutamine dose) are derived [21]. From [21], it is observed that the baseline cardiac ejection fraction (BEF) is inversely correlated with the DOSE $(\mathrm{P}=0.0255)$, indicating that the BEF decreases as the amount of dobutamine dose increases. From [21], it is observed that the cardiac ejection fraction on dobutamine dose (DOBEF) is directly correlated with DOSE $(\mathrm{P}=0.0110)$, indicating that DOBEF increases as the amount of DOSE increases. In addition, the variance of DOBEF is inversely correlated with the dobutamine dose (DD) at maximum double product (DOBDOSE) $(\mathrm{P}=0.0015)$, indicating that DOBEF variance increases as the DOBDOSE decreases. These results are displayed in Table 1.

\section{Effects of dobutamine dose on heart rates (based on heart rate models)}

The effects of dobutamine dose on heart rates (basal, peak and maximum) are derived [20]. It is observed that the mean of basal heart rate (BHR) is inversely correlated with the DPMAXDO $(\mathrm{P}<0.0001)$, indicating that the BHR increases as the DPMAXDO decreases. Moreover, the mean BHR is inversely correlated with the DOBDOSE $(\mathrm{P}=0.0740)$, indicating that the BHR increases as the DOBDOSE decreases. From [20], it is observed that the mean of peak heart rate $(\mathrm{PHR})$ is directly correlated with the DPMAXDO $(\mathrm{P}<0.0001)$, indicating that the PHR increases as the DPMAXDO increases. But the variance of PHR is inversely correlated with the DOBDOSE $(\mathrm{P}<0.0001)$, indicating that the PHR variance increases as the DOBDOSE decreases. From [20], it is observed that the mean of maximum heart rate (MHR) is directly correlated with the DPMAXDO $(\mathrm{P}<0.0001)$, indicating that the MHR increases as the DPMAXDO increases. Also, the variance of MHR is directly correlated with the DOBDOSE $(\mathrm{P}<0.0010)$, indicating that the MHR variance increases as the DOBDOSE increases. These results are reproduced in Table 1 .

\section{Dobutamine dose analysis, results and interpretations}

Dobutamine dose analysis: Dobutamine dose of the DSE data set $[14,15,22]$ is considered as the response variable, and the remaining others (factors/variables) are treated as the explanatory variables. The response dobutamine dose (DOSE) is positive, continuous, heteroscedastic and gamma distributed response variable, so the DOSE has been modeled based on the joint generalized linear gamma models [16-18]. Based on the smallest Akaike information criterion (AIC) value in each class, the final fitted models have been selected. It is noted that AIC selects a model which minimizes the squared error loss and predicted additive errors [23]. The results of joint gamma fitted models of DOSE are given in Table 1 (based on the smallest AIC=2578.353). The included effects of DOSE models are significant (Table 1). The diagnostic plots (normal probability and absolute residual plots) of the fitted gamma models have been examined in Figure 1.

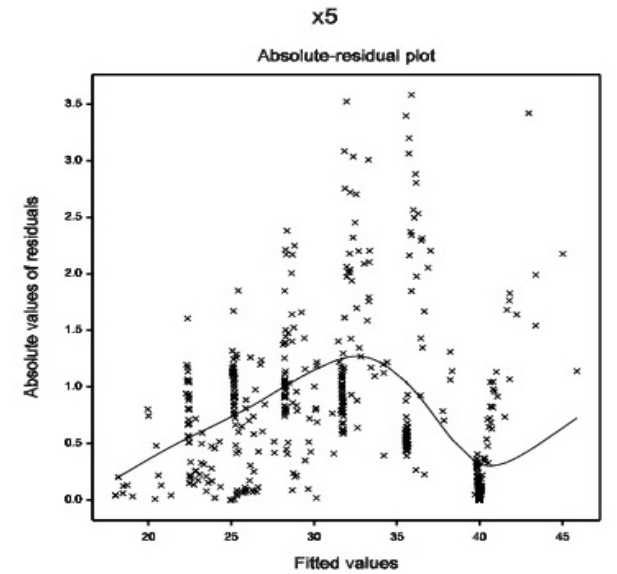

$1(\mathrm{a})$

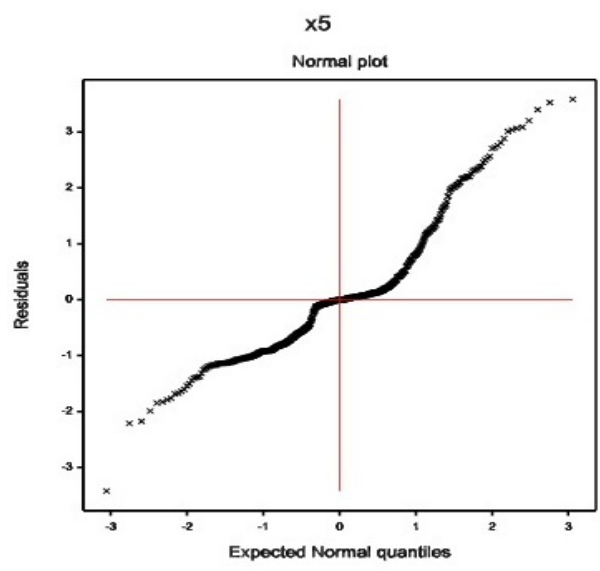

$1(\mathrm{~b})$

Figure 1: For the gamma fitted DOSE models (Table 1) (a) Absolute residuals plot with respect to fitted values; (b) The normal probability plot of the mean model. 
Figure 1(a) reveals the absolute residuals plot of the gamma fitted models of DOSE (Table 1), with respect to fitted values. It is almost flat with the running means except the left and right tails. The left tail is decreasing as the smallest absolute residual value is located at the left extreme, while the right tail is increasing as a larger absolute residual value is located at the right extreme. Figure 1(b) shows the normal probability plot of the gamma fitted mean model of DOSE (Table 1). Note that Figure 1(b) does not reveal any systematic departure of lack of fit. Both the figures (Figures 1a and $1 \mathrm{~b}$ ) reveal that the gamma fit is appropriate for the response DOSE.

\section{Results of Dobutamine Dose Analysis}

Table 1 displays the DOSE analysis results for the mean and variance models. Mean DOSE is negatively associated with the double product of SBP and PKHR (DP) $(\mathrm{P}<0.0001)$, while it is positively associated with the DPMAXDO $(\mathrm{P}<0.0001)$, or DOBDOSE $(\mathrm{P}<0.0001)$. Variance of DOSE is negatively associated with the SBP $(\mathrm{P}<0.0001)$, or DOBDOSE $(\mathrm{P}<0.0001)$, or SEX $(\mathrm{P}=0.0190)$, or new myocardial infraction (new $\mathrm{MI})$ $(\mathrm{P}=0.0010)$, or DEATH $(\mathrm{P}=0.0730)$, while it is positively associated with the maximum blood pressure $(\mathrm{MBP})(\mathrm{P}<0.0001)$, or positive stress on echocardiogram (ECDG) (posSE) $(\mathrm{P}=0.0110)$, or history of MI (hxof MI) $(\mathrm{P}=0.0960)$.

\section{Effects of dobutamine dose on cardiac parameters and events}

The dobutamine dose (DOSE) analysis results are reported as in above, and their summarized form is displayed in Table 1. The following interpretations can be drawn from Table 1.

- The mean DOSE is inversely correlated with the double product of SBP and PKHR (DP) $(\mathrm{P}<0.0001)$, indicating that as the DOSE increases, the DP decreases.

- The mean DOSE is directly correlated with the DPMAXDO $(\mathrm{P}<0.0001)$, indicating that as the DOSE increases, the DPMAXDO increases.

- The mean DOSE is directly correlated with the DOBDOSE $(\mathrm{P}<0.0001)$, indicating that as the DOSE increases, the DOBDOSE increases.

- The variance of DOSE is inversely correlated with the SBP $(\mathrm{P}<0.0001)$, indicating that the DOSE variance increases as the SBP decreases.

- The variance of DOSE is inversely correlated with the DOBDOSE $(\mathrm{P}<0.0001)$, indicating that the DOSE variance increases as the DOBDOSE decreases.

- The variance of DOSE is inversely correlated with the SEX (male $=0$, female $=1) \quad(\mathrm{P}=0.0190)$, indicating that the DOSE variance is higher for males than females.

- The variance of DOSE is inversely correlated with the new myocardial infraction (MI) $(\mathrm{y}=0, \mathrm{n}=1)($ new $\mathrm{MI})(\mathrm{P}=0.0010)$, indicating that the DOSE variance is higher for the cardiac patients having new MI than the patients without new MI.

- The variance of DOSE is inversely correlated with the death $(\mathrm{y}=0, \mathrm{n}=1)(\mathrm{DEATH})(\mathrm{P}=0.0730)$, indicating that the DOSE variance is higher for the cardiac patients who are close to death than the survived patients.

- The variance of DOSE is directly correlated with the maximum blood pressure $(\mathrm{MBP})(\mathrm{P}<0.0001)$, indicating that the DOSE variance increases as the $\mathrm{MBP}$ increases.
- The variance of DOSE is directly correlated with the positive stress on ECDG $(y=0, n=1)$ (posSE) $(\mathrm{P}=0.0110)$, indicating that the DOSE variance is higher for the cardiac patients without posSE than the patients with posSE.

- The variance of DOSE is directly correlated with the history of MI ( $y=0, n=1)$ (hxof MI) $(\mathrm{P}=0.0960)$, indicating that the DOSE variance is higher for the cardiac patients without hxof MI than the patients with hxof MI.

\section{Discussion and Conclusion}

The report focuses the effects of dobutamine dose on the cardiac parameters and cardiac events based on probabilistic gamma joint generalized linear models of cardiac parameters and dobutamine dose. The effects of dobutamine dose are listed in Table 1. The present report has derived many effects of dobutamine dose which are not shown in earlier reports [8-13].

In the report, it is observed that mean SBP and ejection fraction on dobutamine dose are directly correlated with the dobutamine dose, while the baseline ejection fraction is inversely associated with the DOSE. Mean SBP and mean BBP are inversely associated with the DPMAXDO, while mean MBP is directly associated with the DPMAXDO. Only the variance of BBP is directly correlated with the DPMAXDO. Mean of PKHR and MAXHR are directly associated with the DPMAXDO, while the mean of BHR is inversely associated with the DPMAXDO. Note that mean of BHR and variance of PKHR are inversely associated with the DOBDOSE, while the variance of MAXHR is directly associated with the DOBDOSE. Mean dobutamine dose is directly associated with the DPMAXDO and DOBDOSE, while it is inversely associated with the double product of SBP and PKHR (DP). Variance of dobutamine dose is inversely associated with the SBP, DOBDOSE, SEX, DEATH, new MI, while it is positively associated with MBP, posSE, hxof MI.

The above results are derived based on a data set given [14]. Further studies are required to get more results for different DSE data sets. These results may be helpful to the medical practitioners. Based on the derived role of dobutamine dose, medical practitioners are advised to select the appropriate dobutamine dose to the cardiac patients. In addition, cardiac parameters and events of the patients are to be examined after applying the dobutamine dose. It is not safe always.

\section{References}

1. Tuttle RR, Mills J (1973) Derives de la dopamine. Belgium Patent no. 798. p: 51

2. Tuttle RR, Mills J (1975) Dobutamine: Development of a new catecholamine to selectively increase cardiac contractility. Circ Res 36: p185.

3. Kraft-Hunter F, Hinds JE (1973) Cardiac dynamic effects of increasing myocardial contractility without changing heart rate, mean aortic pressure, and end diastolic volume in conscious instrumented dogs. Fed Proc 32: 343

4. Marcovitz PA, Armstrong WF (1992) Accuracy of dobutamine stress echocardiography in detecting coronary artery disease. Am J Cardiol 69: 12691273.

5. Davila-Roman VG, Waggoner AD, Sicard GA, Geltman EM, Schechtman KB et al. (1993) Dobutamine stress echocardiography predicts surgical outcome in patients with an aortic aneurysm and peripheral vascular disease. $\mathrm{J} \mathrm{Am} \mathrm{Coll}$ Cardiol 21: 957-963.

6. Secknus MA, Marwick TH (1997) Evolution of dobutamine echocardiography protocols and indications: Safety and side effects in 3,011 studies over 5 years J Am Coll Cardiol 29: 1234-1240.

7. Konishi T, Koyama T, Aoki T, Yada T, Futagami Y et al. (1990) Radionuclide assessment of left ventricular function during dobutamine infusion in patients with coronary artery disease: Comparison with ergometer exercise. Clin Cardio 13: $183-188$ 
Citation: Das RN (2018) The Role of Dobutamine Dose on the Cardiac Parameters . Cardiovasc Pharm Open Access 7: 238. doi: 10.4172/23296607.1000238

Page 5 of 5

8. Sawada SG, Segar DS, Ryan T, Brown SE, Dohan AM, et al. (1991) Echocardiographic detection of coronary artery disease during dobutamine infusion. Circulation 83: 1605-1614.

9. Mertes H, Sawada SG, Ryan T, Segar DS, Kovacs R, et al. (1993) Symptoms, adverse effects, and complications associated with dobutamine stress chocardiography experience in 1118 patients. Circulation 88: 15-19.

10. Leier CV, Webel J, Bush CA (1977) The cardiovascular effects of the continuous infusion of dobutamine in patients with severe cardiac failure. Circulation 56: 468-472.

11. Mebazaa A, Nieminen MS, Packer M, Cohen-Solal A, Kleber FX, et al. (2007) Levosimendan vs. Dobutamine for patients with acute decompensated heart failure The SURVIVE randomized trial. Journal of American Medical Association 297: 1883-1891.

12. Morelli A, Ertmer C, Lange M, Dunser M, Rehberg S, et al. (2008) Effects of short-term simultaneous infusion of dobutamine and terlipressin in patients with septic shock: The DOBUPRESS study. British Journal of Anaesthesia 100: 494-503.

13. Levy B, Nace L, Bollaert PE, Dousset B, Mallie JP, et al. (1999) Comparison of systemic and regional effects of dobutamine and dopexamine in norepinephrinetreated septic shock. Intensive Care Med 25: 942-948.

14. Krivokapich J, Child JS, Walter D, Garfinkel A (1999) Prognostic value of dobutamine stress echocardiography in predicting cardiac events in patients with known or suspected coronary artery disease. Journal of the American College of Cardiology 33: 708-716.

15. Das RN (2016) Hypertension risk factors who underwent dobutamine stress echocardiography. Interventional Cardiology: Open Access 8: 595-605.

16. Lee Y, Nelder JA, Pawitan Y (2006) Generalized Linear Models with Random Effects (Unified Analysis via H-likelihood). Chapman \& Hall, London.

17. Das RN, Lee $Y$ (2010) Analysis strategies for multiple responses in quality improvement experiments. Int J Qual Engineering Techno 1: 395-409.

18. Das RN, Park JS (2012) Discrepancy in regression estimates between lognormal and gamma: Some case studies. J Applied Statistics 39: 97-111.

19. Firth D (1988) Multiplicative errors: log-normal or gamma. JR Statist Soc 50 266-268.

20. Das RN, Lee Y, Mukhopadhyay BB (2017) The basal, peak and maximum heart rate determinants of the cardiac patients who underwent dobutamine stress echocardiography. Mathews Journal of Cardiology 2: 13.

21. Das RN (2017) Determinants of cardiac ejection fraction for the patients with dobutamine stress echocardiography. Epidemiology 7: 307.

22. Das RN (2016) Dobutamine dose: What are its effects. Interventional Cardiology J 2: 1-3.

23. Hastie T, Tibshirani R, Friedman J (2001) The Elements of Statistical Learning Springer-Verlag, NewYork. 